An Introduction to Complex Analysis 

Ravi P. Agarwal • Kanishka Perera

Sandra Pinelas

\section{An Introduction to Complex Analysis}

照 Springer 
Ravi P. Agarwal

Department of Mathematics

Florida Institute of Technology

Melbourne, FL 32901, USA

agarwal@fit.edu

Sandra Pinelas

Department of Mathematics

Azores University, Apartado 1422

9501-801 Ponta Delgada, Portugal

sandra.pinelas@clix.pt

\author{
Kanishka Perera \\ Department of Mathematical Sciences \\ Florida Institute of Technology \\ Melbourne, FL 32901, USA \\ kperera@fit.edu
}

ISBN 978-1-4614-0194-0

e-ISBN 978-1-4614-0195-7

DOI 10.1007/978-1-4614-0195-7

Springer New York Dordrecht Heidelberg London

Library of Congress Control Number: 2011931536

Mathematics Subject Classification (2010): M12074, M12007

(C) Springer Science+Business Media, LLC 2011

All rights reserved. This work may not be translated or copied in whole or in part without the written permission of the publisher (Springer Science+Business Media, LLC, 233 Spring Street, New York, NY 10013, USA), except for brief excerpts in connection with reviews or scholarly analysis. Use in connection with any form of information storage and retrieval, electronic adaptation, computer software, or by similar or dissimilar methodology now known or hereafter developed is forbidden. The use in this publication of trade names, trademarks, service marks, and similar terms, even if they are not identified as such, is not to be taken as an expression of opinion as to whether or not they are subject to proprietary rights.

Printed on acid-free paper

Springer is part of Springer Science+Business Media (www.springer.com) 
Dedicated to our mothers:

Godawari Agarwal, Soma Perera, and Maria Pinelas 



\section{Preface}

Complex analysis is a branch of mathematics that involves functions of complex numbers. It provides an extremely powerful tool with an unexpectedly large number of applications, including in number theory, applied mathematics, physics, hydrodynamics, thermodynamics, and electrical engineering. Rapid growth in the theory of complex analysis and in its applications has resulted in continued interest in its study by students in many disciplines. This has given complex analysis a distinct place in mathematics curricula all over the world, and it is now being taught at various levels in almost every institution.

Although several excellent books on complex analysis have been written, the present rigorous and perspicuous introductory text can be used directly in class for students of applied sciences. In fact, in an effort to bring the subject to a wider audience, we provide a compact, but thorough, introduction to the subject in An Introduction to Complex Analysis. This book is intended for readers who have had a course in calculus, and hence it can be used for a senior undergraduate course. It should also be suitable for a beginning graduate course because in undergraduate courses students do not have any exposure to various intricate concepts, perhaps due to an inadequate level of mathematical sophistication.

The subject matter has been organized in the form of theorems and their proofs, and the presentation is rather unconventional. It comprises 50 class tested lectures that we have given mostly to math majors and engineering students at various institutions all over the globe over a period of almost 40 years. These lectures provide flexibility in the choice of material for a particular one-semester course. It is our belief that the content in a particular lecture, together with the problems therein, provides fairly adequate coverage of the topic under study.

A brief description of the topics covered in this book follows: In Lecture 1 we first define complex numbers (imaginary numbers) and then for such numbers introduce basic operations-addition, subtraction, multiplication, division, modulus, and conjugate. We also show how the complex numbers can be represented on the $x y$-plane. In Lecture 2, we show that complex numbers can be viewed as two-dimensional vectors, which leads to the triangle inequality. We also express complex numbers in polar form. In Lecture 3, we first show that every complex number can be written in exponential form and then use this form to raise a rational power to a given complex number. We also extract roots of a complex number and prove that complex numbers cannot be totally ordered. In Lecture 4, we collect some essential definitions about sets in the complex plane. We also introduce stereographic projection and define the Riemann sphere. This 
ensures that in the complex plane there is only one point at infinity.

In Lecture 5, first we introduce a complex-valued function of a complex variable and then for such functions define the concept of limit and continuity at a point. In Lectures 6 and $\mathbf{7}$, we define the differentiation of complex functions. This leads to a special class of functions known as analytic functions. These functions are of great importance in theory as well as applications, and constitute a major part of complex analysis. We also develop the Cauchy-Riemann equations, which provide an easier test to verify the analyticity of a function. We also show that the real and imaginary parts of an analytic function are solutions of the Laplace equation.

In Lectures $\mathbf{8}$ and $\mathbf{9}$, we define the exponential function, provide some of its basic properties, and then use it to introduce complex trigonometric and hyperbolic functions. Next, we define the logarithmic function, study some of its properties, and then introduce complex powers and inverse trigonometric functions. In Lectures 10 and 11, we present graphical representations of some elementary functions. Specially, we study graphical representations of the Möbius transformation, the trigonometric mapping $\sin z$, and the function $z^{1 / 2}$.

In Lecture 12, we collect a few items that are used repeatedly in complex integration. We also state Jordan's Curve Theorem, which seems to be quite obvious; however, its proof is rather complicated. In Lecture 13, we introduce integration of complex-valued functions along a directed contour. We also prove an inequality that plays a fundamental role in our later lectures. In Lecture 14, we provide conditions on functions so that their contour integral is independent of the path joining the initial and terminal points. This result, in particular, helps in computing the contour integrals rather easily. In Lecture 15, we prove that the integral of an analytic function over a simple closed contour is zero. This is one of the fundamental theorems of complex analysis. In Lecture 16, we show that the integral of a given function along some given path can be replaced by the integral of the same function along a more amenable path. In Lecture 17, we present Cauchy's integral formula, which expresses the value of an analytic function at any point of a domain in terms of the values on the boundary of this domain. This is the most fundamental theorem of complex analysis, as it has numerous applications. In Lecture 18, we show that for an analytic function in a given domain all the derivatives exist and are analytic. Here we also prove Morera's Theorem and establish Cauchy's inequality for the derivatives, which plays an important role in proving Liouville's Theorem.

In Lecture 19, we prove the Fundamental Theorem of Algebra, which states that every nonconstant polynomial with complex coefficients has at least one zero. Here, for a given polynomial, we also provide some bounds 
on its zeros in terms of the coefficients. In Lecture 20, we prove that a function analytic in a bounded domain and continuous up to and including its boundary attains its maximum modulus on the boundary. This result has direct applications to harmonic functions.

In Lectures 21 and 22, we collect several results for complex sequences and series of numbers and functions. These results are needed repeatedly in later lectures. In Lecture 23, we introduce a power series and show how to compute its radius of convergence. We also show that within its radius of convergence a power series can be integrated and differentiated term-by-term. In Lecture 24, we prove Taylor's Theorem, which expands a given analytic function in an infinite power series at each of its points of analyticity. In Lecture 25, we expand a function that is analytic in an annulus domain. The resulting expansion, known as Laurent's series, involves positive as well as negative integral powers of $\left(z-z_{0}\right)$. From applications point of view, such an expansion is very useful. In Lecture 26, we use Taylor's series to study zeros of analytic functions. We also show that the zeros of an analytic function are isolated. In Lecture 27, we introduce a technique known as analytic continuation, whose principal task is to extend the domain of a given analytic function. In Lecture 28, we define the concept of symmetry of two points with respect to a line or a circle. We shall also prove Schwarz's Reflection Principle, which is of great practical importance for analytic continuation.

In Lectures 29 and 30, we define, classify, characterize singular points of complex functions, and study their behavior in the neighborhoods of singularities. We also discuss zeros and singularities of analytic functions at infinity.

The value of an iterated integral depends on the order in which the integration is performed, the difference being called the residue. In Lecture 31, we use Laurent's expansion to establish Cauchy's Residue Theorem, which has far-reaching applications. In particular, integrals that have a finite number of isolated singularities inside a contour can be integrated rather easily. In Lectures 32-35, we show how the theory of residues can be applied to compute certain types of definite as well as improper real integrals. For this, depending on the complexity of an integrand, one needs to choose a contour cleverly. In Lecture 36, Cauchy's Residue Theorem is further applied to find sums of certain series.

In Lecture 37, we prove three important results, known as the Argument Principle, Rouché's Theorem, and Hurwitz's Theorem. We also show that Rouché's Theorem provides locations of the zeros and poles of meromorphic functions. In Lecture 38, we further use Rouché's Theorem to investigate the behavior of the mapping $f$ generated by an analytic function $w=f(z)$. Then we study some properties of the inverse mapping $f^{-1}$. We also discuss functions that map the boundaries of their domains to the 
boundaries of their ranges. Such results are very important for constructing solutions of Laplace's equation with boundary conditions.

In Lecture 39, we study conformal mappings that have the anglepreserving property, and in Lecture $\mathbf{4 0}$ we employ these mappings to establish some basic properties of harmonic functions. In Lecture 41, we provide an explicit formula for the derivative of a conformal mapping that maps the upper half-plane onto a given bounded or unbounded polygonal region. The integration of this formula, known as the Schwarz-Christoffel transformation, is often applied in physical problems such as heat conduction, fluid mechanics, and electrostatics.

In Lecture 42, we introduce infinite products of complex numbers and functions and provide necessary and sufficient conditions for their convergence, whereas in Lecture 43 we provide representations of entire functions as finite/infinite products involving their finite/infinite zeros. In Lecture 44, we construct a meromorphic function in the entire complex plane with preassigned poles and the corresponding principal parts.

Periodicity of analytic/meromorphic functions is examined in Lecture 45. Here, doubly periodic (elliptic) functions are also introduced. The Riemann zeta function is one of the most important functions of classical mathematics, with a variety of applications in analytic number theory. In Lecture 46, we study some of its elementary properties. Lecture 47 is devoted to Bieberbach's conjecture (now theorem), which had been a challenge to the mathematical community for almost 68 years. A Riemann surface is an ingenious construct for visualizing a multi-valued function. These surfaces have proved to be of inestimable value, especially in the study of algebraic functions. In Lecture 48, we construct Riemann surfaces for some simple functions. In Lecture 49, we discuss the geometric and topological features of the complex plane associated with dynamical systems, whose evolution is governed by some simple iterative schemes. This work, initiated by Julia and Mandelbrot, has recently found applications in physical, engineering, medical, and aesthetic problems; specially those exhibiting chaotic behavior.

Finally, in Lecture 50, we give a brief history of complex numbers. The road had been very slippery, full of confusions and superstitions; however, complex numbers forced their entry into mathematics. In fact, there is really nothing imaginary about imaginary numbers and complex about complex numbers.

Two types of problems are included in this book, those that illustrate the general theory and others designed to fill out text material. The problems form an integral part of the book, and every reader is urged to attempt most, if not all of them. For the convenience of the reader, we have provided answers or hints to all the problems. 
In writing a book of this nature, no originality can be claimed, only a humble attempt has been made to present the subject as simply, clearly, and accurately as possible. The illustrative examples are usually very simple, keeping in mind an average student.

It is earnestly hoped that An Introduction to Complex Analysis will serve an inquisitive reader as a starting point in this rich, vast, and ever-expanding field of knowledge.

We would like to express our appreciation to Professors Hassan Azad, Siegfried Carl, Eugene Dshalalow, Mohamed A. El-Gebeily, Kunquan Lan, Radu Precup, Patricia J.Y. Wong, Agacik Zafer, Yong Zhou, and Changrong Zhu for their suggestions and criticisms. We also thank Ms. Vaishali Damle at Springer New York for her support and cooperation.

Ravi P Agarwal

Kanishka Perera

Sandra Pinelas 



\section{Contents}

Preface

vii

1. Complex Numbers I 1

2. Complex Numbers II $\quad 6$

3. Complex Numbers III 11

4. Set Theory in the Complex Plane $\mathbf{2 0}$

5. Complex Functions $\quad 28$

6. Analytic Functions I 37

7. Analytic Functions II 42

8. Elementary Functions I 52

9. Elementary Functions II $\quad 57$

10. Mappings by Functions I 64

11. Mappings by Functions II $\quad 69$

12. Curves, Contours, and Simply Connected Domains 77

13. Complex Integration 83

$\begin{array}{ll}\text { 14. Independence of Path } & 91\end{array}$

15. Cauchy-Goursat Theorem 96

16. Deformation Theorem 102

17. Cauchy's Integral Formula 111

18. Cauchy's Integral Formula for Derivatives $\mathbf{1 1 6}$

19. The Fundamental Theorem of Algebra $\mathbf{1 2 5}$

20. Maximum Modulus Principle 132

21. Sequences and Series of Numbers 138

22. Sequences and Series of Functions 145

23. Power Series 151

24. Taylor's Series 159

25. Laurent's Series 169 
26. Zeros of Analytic Functions

27. Analytic Continuation

28. Symmetry and Reflection

29. Singularities and Poles I

30. Singularities and Poles II

31. Cauchy's Residue Theorem

32. Evaluation of Real Integrals by Contour Integration I

33. Evaluation of Real Integrals by Contour Integration II

34. Indented Contour Integrals

35. Contour Integrals Involving Multi-valued Functions

36. Summation of Series

37. Argument Principle and Rouché and Hurwitz Theorems

38. Behavior of Analytic Mappings

39. Conformal Mappings

40. Harmonic Functions

41. The Schwarz-Christoffel Transformation

42. Infinite Products

43. Weierstrass's Factorization Theorem

44. Mittag-Leffler Theorem

45. Periodic Functions

46. The Riemann Zeta Function

47. Bieberbach's Conjecture 308

48. The Riemann Surfaces 312

49. Julia and Mandelbrot Sets 316

50. History of Complex Numbers 321

References for Further Reading $\quad 327$

Index 